\title{
Chapter 3 \\ Historical Maritime Search and Rescue Incident Data Analysis
}

\author{
Mark A. Stoddard and Ronald Pelot
}

\begin{abstract}
Since the 1980s the Canadian Coast Guard has maintained a database of maritime search and rescue (SAR) incidents involving response assets and personnel. This information is stored in a national database known as the Search and Rescue Program Information Management System (SISAR). SISAR contains a spatiotemporal record for all serious incidents that occur within Canada's coastal search and rescue area. In addition to providing a record of all response operations, it provides a rich historical dataset for analysts to use to support a wide range of decisionmaking applications. In this chapter we illustrate the use of SISAR incident data to identify and visualize temporal and spatial patterns in the maritime SAR incident data. Temporal phenomena were examined at three temporal scales: yearly, monthly, and hourly. Spatial phenomena were examined using the spatial location and density of incidents. Several useful visualizations to explore and exploit SISAR data are provided. Lastly, we provide a short discussion of several topics relevant to SAR incident analysis, including (1) under-reporting in incident databases, (2) sharing of national SAR incident data, and (3) linking environmental conditions and accident data to add context to historical SAR incidents and to improve SAR response time estimation.
\end{abstract}

Keywords D3 - Data analytics · Incident analysis - Search and rescue Visualization

M. A. Stoddard $(\bowtie) \cdot$ R. Pelot

Department of Industrial Engineering, Dalhousie University, Halifax, NS, Canada

e-mail: Mark@dal.ca; ronald.pelot@dal.ca 


\subsection{Introduction}

In Canada, search and rescue (SAR) is one of the primary responsibilities of the Canadian Coast Guard (CCG). The CCG's SAR programme is needed largely due to the size of Canada's coastal search and rescue area $\left(\sim 5.3\right.$ million $\left.\mathrm{km}^{2}\right)$, consisting of both extensive inland waterways and open ocean (Government of Canada 2019c). Through the effective use of SAR resources, the CCG responds to approximately 6000 maritime incidents per year (Government of Canada 2019a). As with most emergency response services, such as police and fire, incident reporting and recordkeeping is a critical part of the response. Following each response operation, CCG incident reports and logs are entered in a database known as the Search and Rescue Program Information Management System (SISAR). SISAR is a web-based database that integrates all regional response data into one national system. SISAR incident data is one of the primary data sources used to capture statistics relating to SAR cases to help inform demand for programme services and the achievement of outcomes (Government of Canada 2019a).

This chapter provides an overview of the CCG SISAR database, a short background of spatiotemporal analysis of SAR incident data, and several interactive web visualizations that have been developed to specifically explore and exploit spatial and temporal trends in available SISAR incident data. Section 3.2 provides an overview of spatiotemporal analysis of SAR incident data and related research. Section 3.3 introduces the CCG SISAR database and the key elements that were used to construct the visual analytics presented in Sect. 3.4 and applied in Sect. 3.5. Section 3.6 discusses the results from the analysis completed in Sect. 3.5 and potential future work. Lastly, Sect. 3.7 provides some concluding remarks.

\subsection{Spatiotemporal Analysis of Search and Rescue Incident Data}

SAR incident data provides a rich multivariate spatiotemporal dataset that can support a wide range of analyses. The availability of spatial attribute data enables the use of spatial statistics and geo-referenced data processing techniques (Shahrabi 2003), while temporal attribute data allows for the use of time series analysis and the study of temporal phenomena and trends (Malik et al. 2012). This form of data has been widely used by the CCG and academic researchers to examine issues related to maritime SAR resource planning and evaluation, such as assessing manning levels (Marven et al. 2007; Government of Canada 2019b) and identifying critical locations for permanent SAR resources (Akbari et al. 2017; Akbari et al. 2018; Pelot and Plummer 2008).

Shahrabi (2003) completed an early example of spatial and temporal analysis of fishing and marine traffic incidents off the coast of Nova Scotia using available SISAR data. The emphasis of this work was on developing a better understanding 
of the location of fishing incidents and their time of occurrence. Using a geographic information system (GIS) and a variety of spatial statistical methods, such as kernel density estimation and hierarchical clustering methods, Shahrabi was able to identify areas of higher risk to fisherman off the coast of Nova Scotia. Furthermore, by analysing temporal incident attributes, the author was able to highlight times of the year where the likelihood of a SAR event was elevated. Pelot and Plummer (2008) expanded on this work to provide a complete assessment of the risk in the Atlantic coastal zone, largely based on maritime traffic modelling and historical SAR incident data. A similar SAR incident analysis was completed by Goerlandt, Venäläinen, and Siljander (2015), focusing on SAR incidents in the Finnish part of the Gulf of Finland. The authors successfully combined historical SAR incident analysis and information derived from GIS methods to perform a risk-informed capacity evaluation of voluntary SAR services. Their method relied on SAR incident data, meteorological data, and Search and Rescue Unit (SRU) data to derive several quantitative risk indicators. These risk indicators were used to assess the SAR response performance for recreational boating incidents.

In addition to the use of SAR incident data to support spatiotemporal analysis, it can also be used to support decision-making. Marven, Canessa, and Keller (2007) show how SAR incident data can be used to support SAR resource planning. Using exploratory spatial data analysis (ESDA) methods suitable for point pattern analysis, these authors proposed several resource allocation modelling approaches based on historical incident data that utilized linear programming, Monte Carlo simulation, and process simulation. More recently, Akbari, Eiselt, and Pelot (2017) proposed a goal programming multi-objective model for locating and allocating maritime SAR vessels. The model considered three objectives for the maritime SAR location-allocation problem: (1) primary coverage, (2) backup coverage, and (3) mean access/response time. When considering historical SAR incident data and the current arrangement of SAR vessel by type and location in their study area (Atlantic Canada), it was shown that substantial improvements, in terms of access time and coverage, may be possible by using the optimal location-allocation solution from their multi-objective model.

Malik et al. (2012) proposed a visual analytic process for maritime response, asset allocation, and risk assessment. The resulting visual analytic system, Coast Guard Search and Rescue Visual Analytics (cgSARVA), was developed to exploit the US Coast Guard (USCG) historical response operations database, covering operations in the Great Lakes region from 2002 to 2011. cgSARVA allows USCG analysts to visually interact with historical SAR incident data, helping to better understand data quality issues and to effectively perform data exploration and analysis. Recently, cgSARVA became part of the USCG initiated Station Optimization Process (US Department of Homeland Security 2018). This process was meant to analyse USCG boat stations and identify those that could be closed because they provide overlapping and/or unnecessarily duplicative SAR coverage.

Lastly, Sonninen and Goerlandt (2015) have explored the meteorological context of maritime SAR missions in the Gulf of Finland using visual data mining techniques to better understand which SAR incident types occur under challenging wind 
and wave conditions. The researchers associated wind and wave data with incident data from a SAR operations database. The associated data was then used to compare different SAR mission types, as well as the activity of different SAR organizations, during challenging wind and wave conditions. Using visual analytic techniques, the authors were able to identify the densest areas of challenging wind and wave conditions and areas that had the highest density of incidents.

\subsection{Search and Rescue Program Information Management System}

In Canada there are three primary SAR regions, each associated with a Joint Rescue Coordination Centre (JRCC), which are jointly operated by the Department of National Defence (DND) and CCG personnel. The JRCC is responsible for promoting the efficient organization of SAR services and for coordinating the conduct of SAR operations within an associated SAR region (Government of Canada 2019a). Currently, when a JRCC receives a report of a vessel in distress, they dispatch the most appropriate SAR resource to provide assistance. Following each SAR event, a new record is added to the SISAR database. SISAR provides a spatiotemporal record of all the serious incidents that required SAR response within Canada's coastal search and rescue area. SISAR was created to provide CCG personnel with easy access to essential information to support SAR planning, management, and operations (Marven et al. 2007).

The incident description fields of the SISAR database are used primarily for CCG internal accounting of events. A unique ID is assigned to each event and is used for all subsequent reporting. Example incident description attributes include the alert method used by the vessel in distress, location of the incident, start and end date time group (DTG), and a text summary of the event provided by the first responders. The incident description data that was used in this study included the incident ID, location, start and end DTG, and severity.

The SAR resource usage fields are used to identify the SAR resource used to respond to the incident and details of the mission. These fields try to capture the nature of the response operation. Fields such as incident severity, distance to the incident, alert time, on-scene time, and distance towed are used to describe CCG resource usage. The SAR resource usage data that was used in this study included the incident severity, incident distance from shore, and alert time. Incident severity is rated by the CCG using a four-point scale, with one being the most severe and four being a false alarm.

The SAR resource deployment fields are used to identify the region, base, and squadron of the SAR response asset. Often this information is associated with a SAR region. Currently, Canada is broken up into three regions: (1) Eastern, (2) Pacific, and (3) Central and Arctic. Each region is then further broken up into 
smaller SAR areas. The smaller SAR areas are used for aggregating and reporting marine incident statistics to support resource allocation planning (Marven et al. 2007). SAR resource deployment data were not used in this study.

The final descriptor, unit assisted field, describes the various characteristics of the vessel that was involved in the incident, including vessel dimensions, flag state, vessel type, class, and the number of persons on board. This information is used by the JRCC to select the most appropriate asset to respond. This information is useful when examining issues related to asset suitability and capacity given the expected unit characteristics that may be encountered during an incident. The only unit assisted data that was used in this study was the vessel length (metres).

\subsection{Visual Analytics for SISAR Data Analysis}

In this section we introduce several useful visualizations that were implemented in Data-Driven Documents (D3) to explore a SISAR dataset containing approximately 36,000 SAR response records. This dataset covers a date range from 2005 to 2013 (excluding 2007, which is not available) and includes records from incidents that have occurred within Canada's coastal SAR areas requiring a physical response by a CCG SAR asset. The use of a multi-year dataset was required to enable the visualizations presented in Sect. 3.4.2. The date range from 2005 to 2013 was arbitrarily chosen because the focus of this study was on the development and potential use of the visual analytics for historical SAR incident analysis presented in this section, rather than the specific results generated by these analytics when used to analyse a dataset.

D3 is an open-source JavaScript library that enables the manipulation of web visualizations, such as charts and graphs, based on underlying data (David and Tauro 2015). D3 accomplishes this by providing a declarative framework for mapping underlying data to visual elements in a web page. This mapping enables the direct inspection and manipulation of a native data representation through user interaction with the web browser (Bostock et al. 2011). Bostock (2019) provides open access to D3 documentation and a large repository of web visualization examples submitted by the D3 user community.

Using D3, each SAR incident is treated as an entity with associated attributes, and this information can be used to visualize the temporal and spatial relationships that exist within the data. To identify temporal phenomena in the data, ordinal classes were used to organize entity temporal attributes by year, month, day, and hour to construct a variety of data visualizations. Figure 3.1 provides a simple hierarchical view of the relationship between SAR incidents and attribute data used in this study. 


\section{ENTITY $\rightarrow$ RELATIONSHIP $\rightarrow$ ATTRIBUTE}

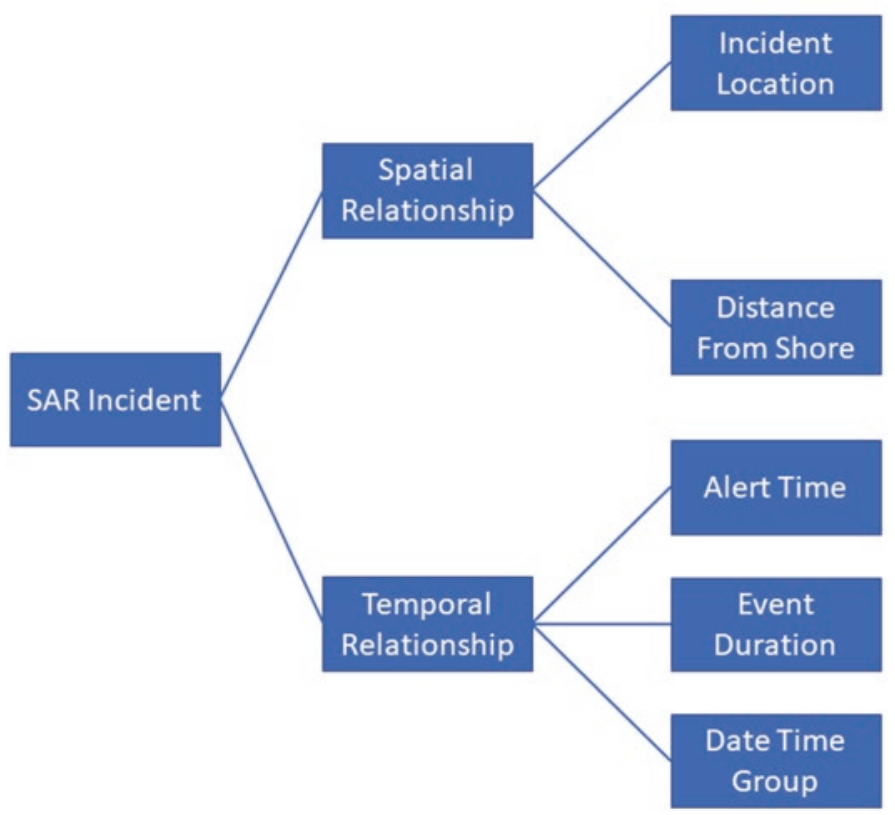

Fig. 3.1 Hierarchical view of entity relationship model

\subsubsection{Interactive SAR Incident Dashboard}

The interactive SAR incident dashboard provides a web visualization for exploring the temporal distribution of SAR incidents contained in the SISAR database. This interactive visualization allows the user to quickly examine the monthly and daily distribution of total incidents from the SISAR dataset using a standard web browser. The data visualized in this section is for calendar year 2013 and contains a total of 4062 SAR incident records. The distribution of monthly total incidents for 2013 is visualized as a bar chart. The dashboard pie chart is used to visualize the weekday distribution of total incidents. A seven-sector pie chart is used to conveniently display the information, with each sector representing a day of the week. The pie chart is initialized using the total number of incidents that occurred on each weekday during the 2013 calendar year. Figure 3.2 shows the initialized interactive incident dashboard visualization using 2013 SAR incident data.

The interactive elements of the SAR incident dashboard are used to enable the exploration of the temporal distribution of SAR incidents in the SISAR. These elements of the dashboard allow the user to rapidly sort and filter the SAR incident data by hovering the mouse pointer over a single bar in the bar chart to select a particular month or hovering over a single sector in the pie chart to select a particular day of 

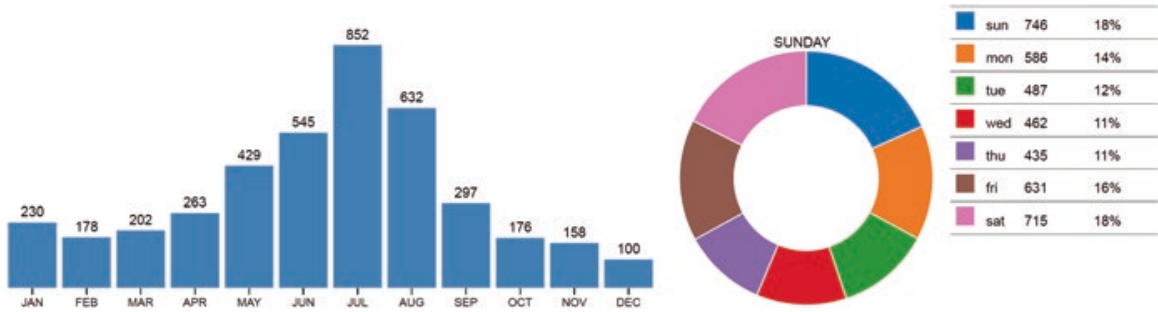

Fig. 3.2 SISAR interactive monthly/weekday SAR incident dashboard for calendar year 2013 (all data for figures and tables in this chapter from Canadian Coast Guard 2017)
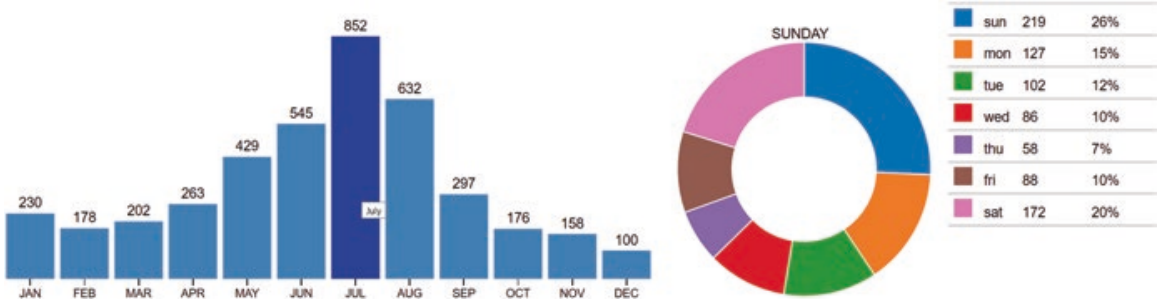

Fig. 3.3 Interactive dashboard result showing the weekday distribution of the total number of incidents that occurred during July 2013

the week. Hovering over these elements of the visualization will automatically render the corresponding analysis result on the web page. Hovering over a single bar in the bar chart will cause the pie chart to render the distribution of monthly total incidents by day of the week. Hovering over a single sector in the pie chart will cause the bar chart to render the distribution of yearly total incidents for a selected day of the week.

Figures 3.3 and 3.4 illustrate the use of the interactive elements of the SAR incident dashboard. Figure 3.3 shows the same monthly distribution of SAR incidents as shown in Fig. 3.2. The month of July has been selected in the bar chart by hovering the mouse over the appropriate bar (highlighted column in bar chart). The result of this user interaction is that the corresponding pie chart automatically renders to show the weekday distribution of incidents for the month of July. Figure 3.4 shows the monthly distribution of total SAR incidents that occurred on Sunday. In this case, Sunday has been selected by hovering the mouse over the sector of the pie chart that corresponds to Sunday. The result of this user interaction is that the bar chart automatically renders to show the monthly distribution of total SAR incidents that occurred on Sunday. 

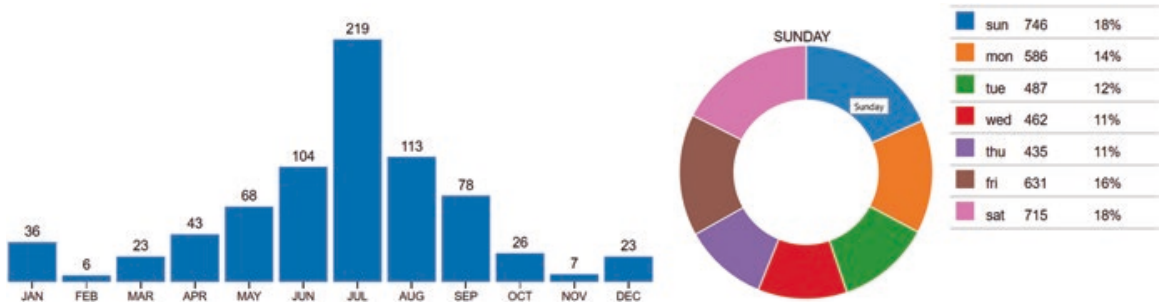

Fig. 3.4 Interactive dashboard result showing the monthly distribution of total incidents that occurred on Sunday during 2013

\subsubsection{Multi-year Monthly Incident Analysis}

The incident dashboard previously described only allows a user to visualize the monthly and weekday distribution of SAR incidents for a single calendar year. In order to satisfy the need to visualize longer-term trends in the SISAR incident data, three multi-year visualizations were used: (1) multi-year monthly incident time series graph, (2) multi-year monthly incident time series chart, and (3) multi-year monthly incident heat map. All three visualizations display the same information, but use three different approaches. Van Wijk and Selow (1999) provide an extensive description of calendar-based visualization of time series data like those presented in this chapter.

\subsubsection{Multi-year Monthly Incident Time Series Graph and Chart}

Using the date and time information associated with a SAR incident enables the visualization of SAR incident data as a time series. Each month was paired with an incident total and plotted. Using simple linear interpolation, a line was constructed that connects the dots that represent the total incidents for a month. Typically, continuous lines are not valid for categorical data and a bar graph should be used. An exception exists when the categorical data is ordered, which is the case in Figs. 3.5 and 3.6 where the data is ordered by calendar month. By overlaying multiple years of data, we can compare the set of line plots to gain insight into multi-year trends. Figure 3.5 shows a multi-series line chart of monthly total incident data from 2005 to 2013. For comparison, Fig. 3.6 shows the same data as a multi-year time series graph. Figure 3.6 more clearly emphasizes the cyclical nature of total monthly SAR incidents over a multi-year period and the exclusion of data from 2007 that was noted at the beginning of this section. 


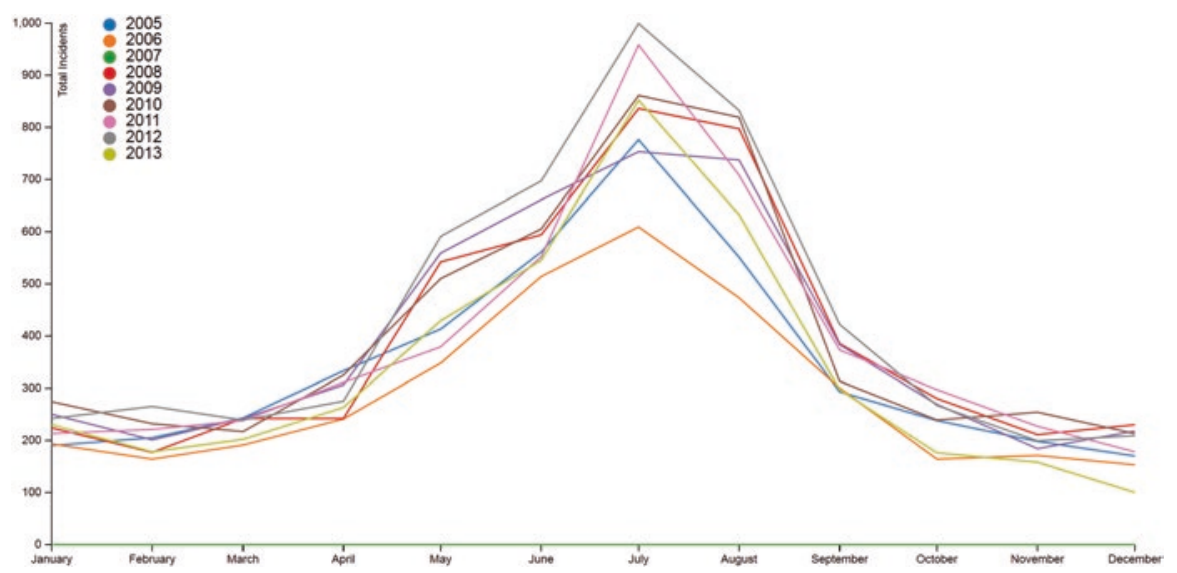

Fig. 3.5 Multi-series line chart of monthly incident totals (2005-2013)

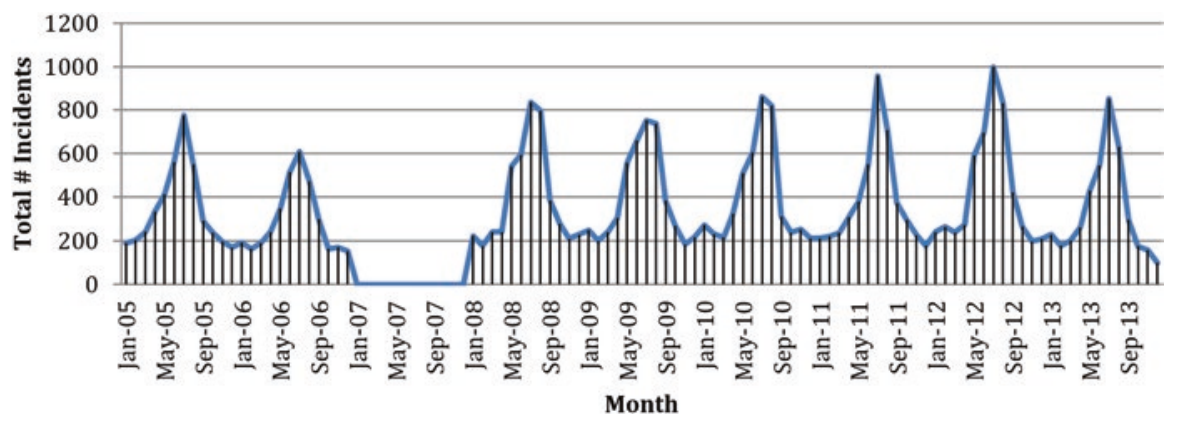

Fig. 3.6 Multi-year time series graph of monthly incident totals (2005-2013)

\subsubsection{Multi-year Monthly Incident Heat Map}

A heat map can most simply be thought of as a two-dimensional representation of data, where colour is used to represent attribute value. The heat map value used for this study was the total number of incidents, and the two dimensions are the month (y-axis) and the year (x-axis). Trends are identified by locating areas of common attribute value (colour) in both dimensions. In Fig. 3.7, darker blue areas are associated with a higher number of total monthly incidents, while the lighter areas represent a lower number of monthly incidents.

\subsubsection{Hourly Total Incident Heat Map}

The hourly total incident heat map allows the user to visualize the 24-hour distribution of SAR incident alert time as a simple circular heat map. The heat map was broken up into 24 sectors, with each sector representing an hour of the day. The 


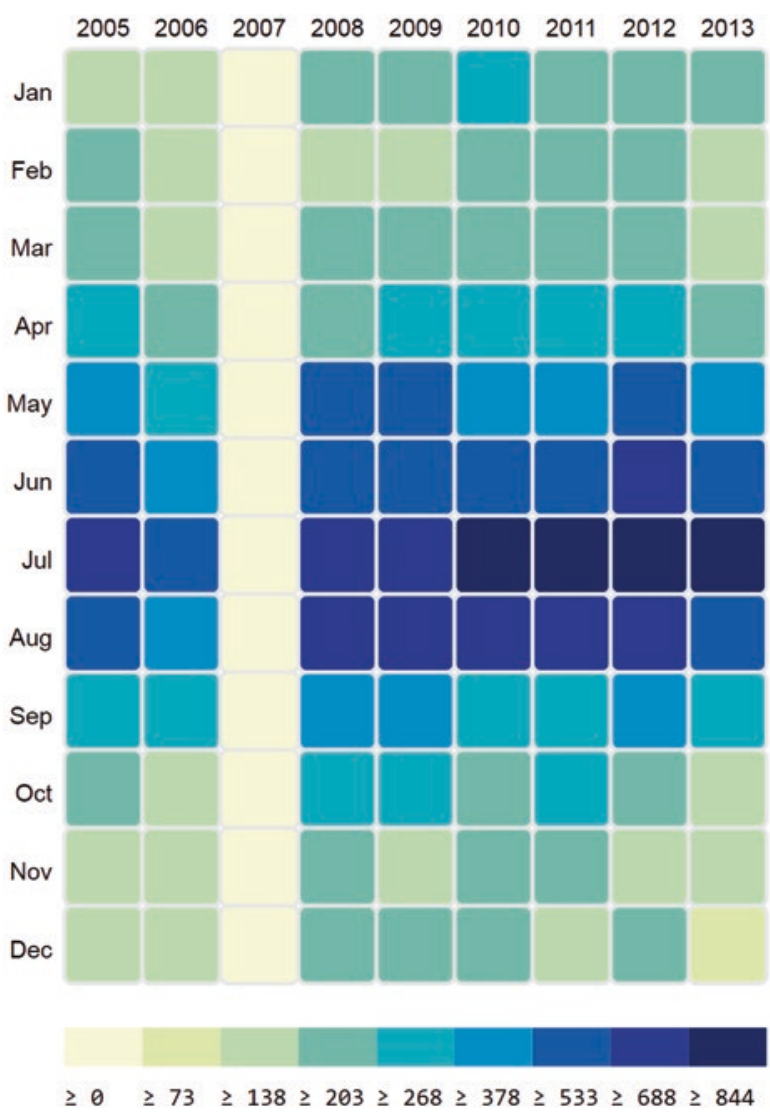

Fig. 3.7 Multi-year monthly total incident heat map (2005-2013)

colour coding was used to represent the total number of SAR incident alerts that were received during each hour of the day, where dark red represents the hour with the maximum number of incidents and dark blue represents the hour with the minimum number of incidents. This visualization allows the user to quickly identify the hours of the day where the greatest number of SAR alerts is expected to be received. Figure 3.8 shows the aggregate hourly distribution of SAR incident alerts from 2005 to 2013.

\subsubsection{Spatial Analysis Map}

The spatial analysis map allows the user to visualize the location of SAR incidents on a map. This visualization allows the user to exploit the spatial proximity and similarity of events to extract meaning (Ware 2004). Figure 3.9 provides a coastline 


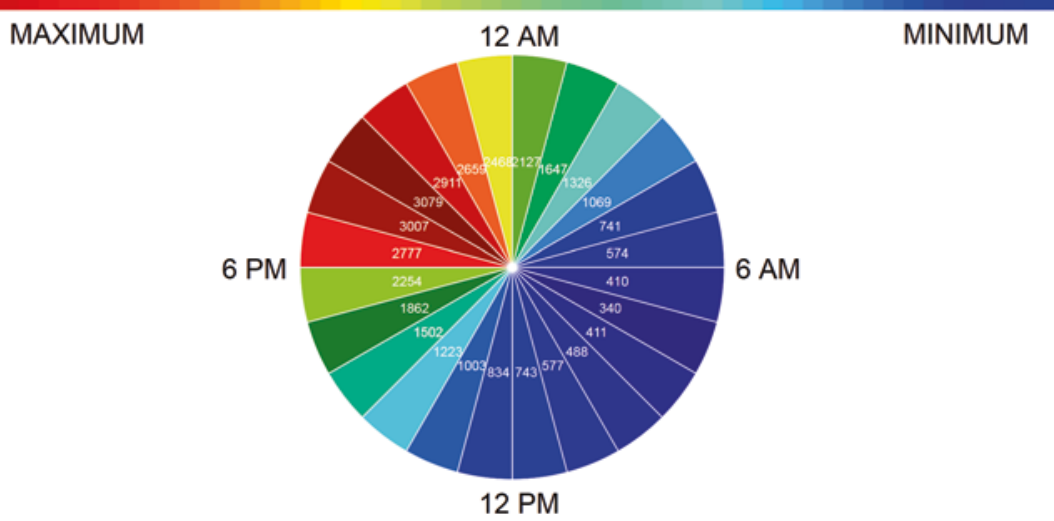

Fig. 3.8 Aggregate hourly total incident heat map of SAR incident alerts from 2005 to 2013

contour to allow the user to reference the incident location to a known geographic area. Two aspects of the spatial analysis map are discussed below, the analysis map and the SISAR data layer.

The analysis map was constructed using a GeoJSON of the Canadian coastline and the D3 default map projection library (Murray 2013). The size and shape of the country being mapped determines the most suitable projection. Since the SISAR data used in this study covers all of Canada, selecting a map projection that could be used to display the location of all incidents is challenging. Maps of very large countries like Canada often appear distorted due to the curvature of the earth. The distortion is minimal over small distances, but for maps of Canada that include the Canadian Arctic, it can be extreme. Since the majority of the SISAR incident data is located below $60^{\circ}$ North, we have chosen to use a standard Albers map projection. The Albers projection is commonly used for land masses that extend in an east-towest orientation, like Canada and the United States (ESRI n.d.). Figure 3.9 shows a zoomed-in map view of Atlantic Canada.

The SISAR data used in this study covers all of Canada's coastal search and rescue area. The dataset contains roughly 36,000 incidents, each with an associated geo-referenced position. By plotting the position of each incident on the analysis map, we can visualize the spatial distribution of incidents. By looking at the spatial proximity among incidents, we can identify areas of higher incident concentration. Shahrabi (2003) provided an excellent example of how this information, when combined with kernel density estimation and hierarchical clustering methods, can be used to identify areas of higher risk. The approach taken in this study was to try and use attribute data from the SISAR dataset to produce a visual analytic to help identify and localize areas of higher risk.

The approach uses the severity of the incident to determine the opacity of the plotted SISAR data. The visual effect is that more severe incidents appear as opaque bright red dots, while false alarms appear almost transparent (Fig. 3.10). The use of 


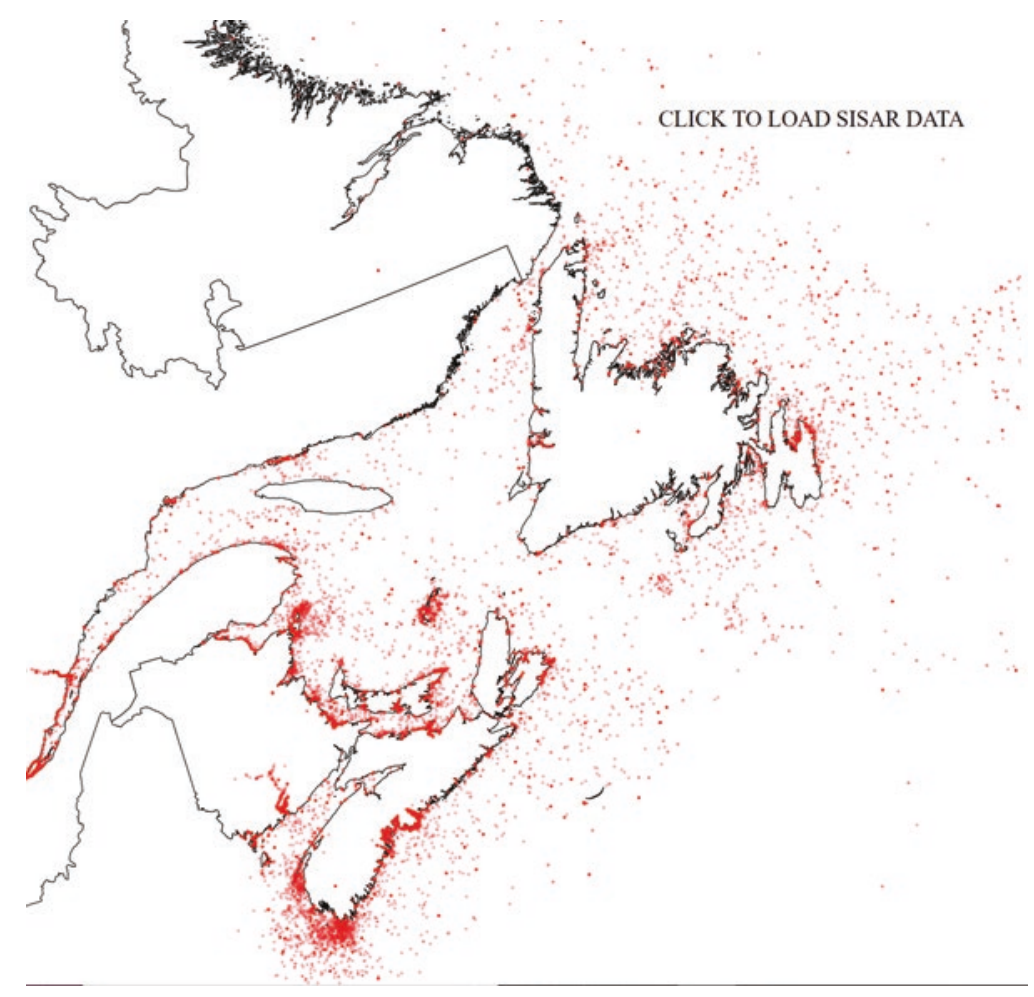

Fig. 3.9 Geospatial analysis map with incident location shown as red point locations

this visual analytics will be discussed in the next section. Equation 3.1 describes how opacity is derived using SAR incident severity data.

$$
\text { Opacity }=\frac{1}{\text { Incident Severity }^{2}}
$$

\subsection{SISAR Data Analysis and Results}

This section showcases a few potential use cases for the visualizations presented in this chapter. Visualizations have been selected that allow an analyst to explore both temporal and spatial trends in the SISAR dataset using a standard web browser. Specifically, three questions are addressed:

1. What was the temporal (by hour, month, and day of week) distribution of the 2013 SAR incidents? 


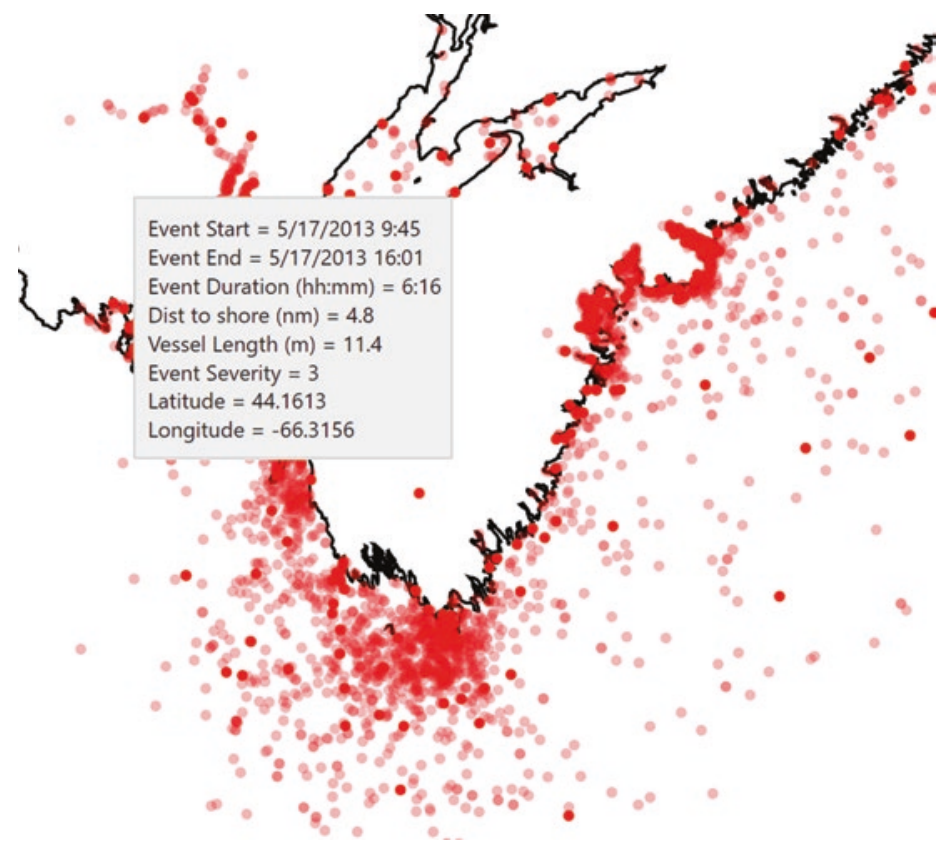

Fig. 3.10 Zoomed-in map view showing the geo-referenced position of incidents plotted as bright red circles with the opacity determined by Eq. 3.1. Hovering the mouse pointer over each georeferenced data point provides the detailed summary of the incident

2. Based on the historical data for all SAR cases, what was the expected annual response case demand broken down by month?

3. Based on the historical data for all SAR cases, what regions show a high concentration of most severe incidents?

\subsubsection{What Is the Temporal Distribution of the Response Case Load?}

The interactive incident dashboard introduced in Sect. 3.4 allows the user to quickly identify monthly and weekday trends in the SISAR data. For any given calendar year in the study period, the user can quickly examine the total number of incidents responded to in a month or on a given weekday. Figure 3.2 showed the 2013 monthly distribution of incidents. A distinct peak in the total number of incidents is easily observed during the summer months (June-August). The peak during the summer months is observed during every year in the SISAR data and was best illustrated by the multi-year time series line chart of monthly incident totals shown in Fig. 3.6. This was likely due to the increase in pleasure boat activity associated with the summer months, previously observed and reported by Malik et al. (2011), Sonninen and Goerlandt (2015), and the Government of Canada (2019a). 


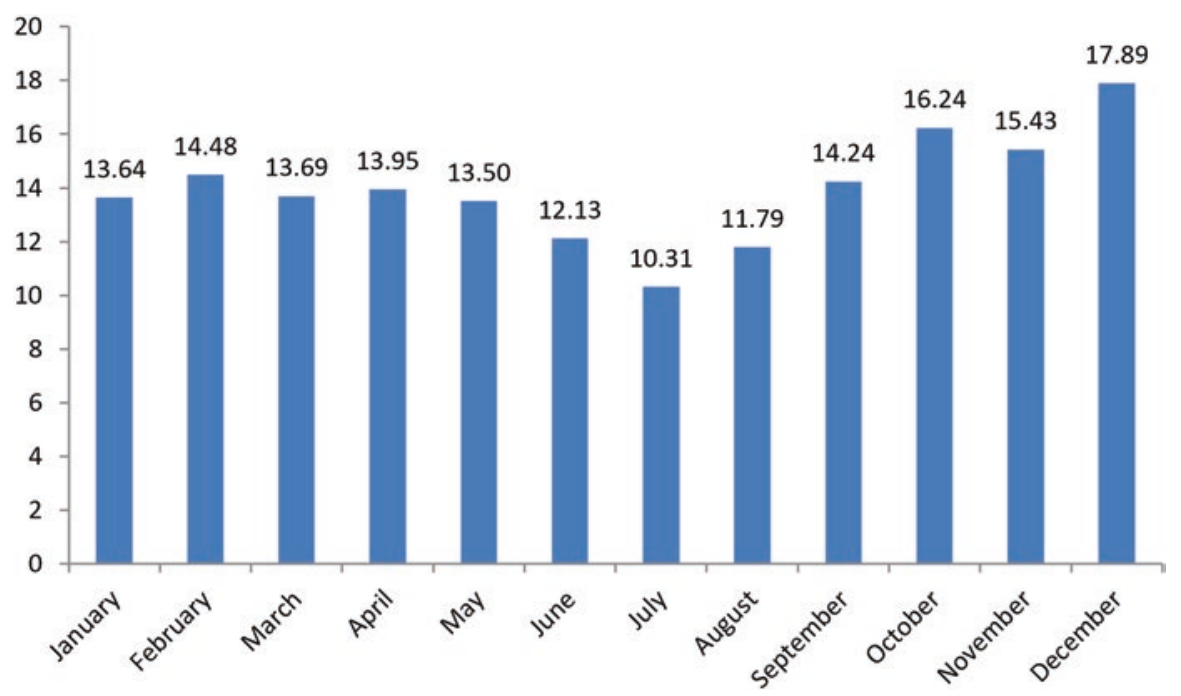

Fig. 3.11 Average SAR incident vessel length (metres) by month (2005-2013)

In an attempt to substantiate this claim using a visual analytic approach, the available SISAR data was processed to produce a bar chart visualization showing the monthly distribution of average vessel length involved in each SAR incident from 2005 to 2013. Figure 3.11 shows a decrease in average vessel length during the summer months, which is likely due to the increase in the number of smaller pleasure boats involved in incidents. Figure 3.12 also shows a decrease in the SAR incident distance from shore during the summer months, which is also likely due to the increased number of pleasure boats on the water.

In addition to the increase in the total number of incidents experienced during the summer months, it was also observed that during the summer months a greater proportion of incidents occurred on the weekend (Saturday and Sunday). For example, a user selecting July will see that the weekend accounted for $46 \%$ of the total number of incidents. Compare this with January where the weekend only accounted for $24 \%$ of the total number of incidents. For comparison, Fig. 3.13 shows the weekday distribution of SAR incidents for July and January.

Lastly, the hourly distribution of SAR incidents was examined. By aggregating all incidents found in the SISAR database, we can generate the hourly total incident heat map discussed in Sect. 3.4.3 and shown in Fig. 3.8. There was an elevated number of incidents between the hours of $6 \mathrm{pm}$ and $11 \mathrm{pm}$. This reporting behaviour is typically associated with pleasure boat activity where a mariner is performing a day trip, where no overnight boating activity is expected (Government of Canada 2003). The SAR incident is triggered by a failure to arrive at the intended destination, return to port on time, or is generally considered overdue (ibid). Malik et al. (2012) also report a very similar hourly distribution of SAR incident case load for the USCG Great Lakes region. 


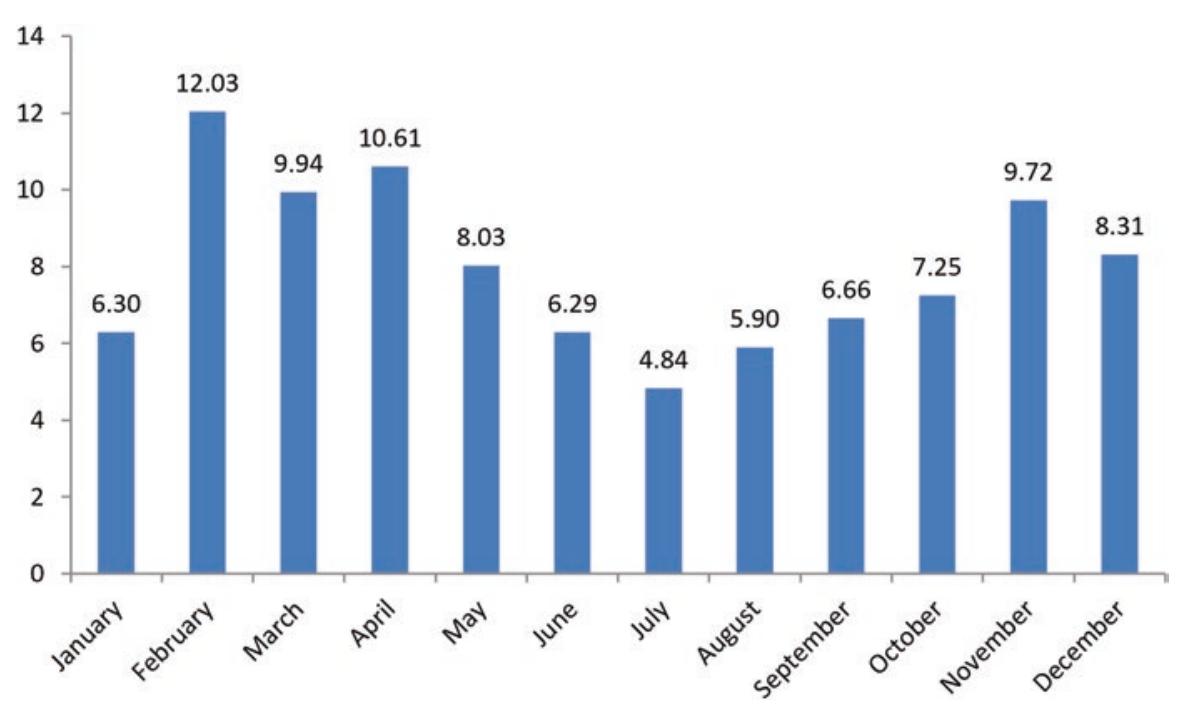

Fig. 3.12 Average SAR incident distance from shore (kilometres) by month (2005-2013)
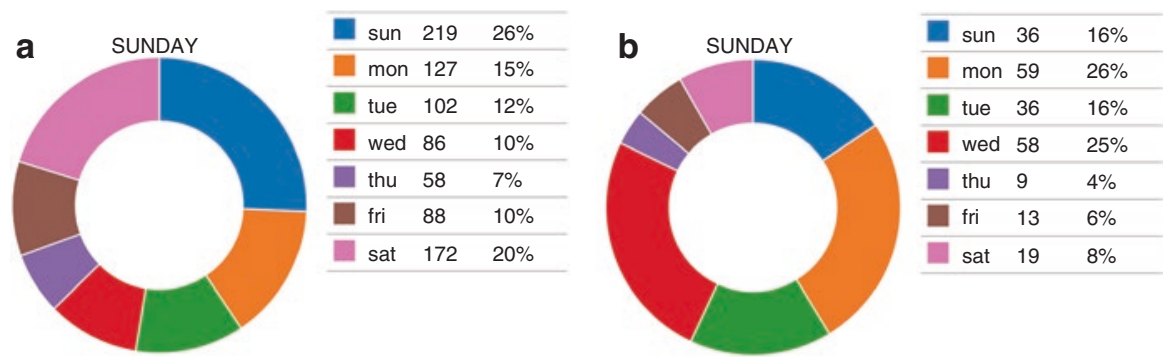

Fig. 3.13 (a) Weekday distribution of July 2013 total SAR incidents, (b) weekday distribution of January 2013 total SAR incidents

\subsubsection{What Is the Expected Annual Response Case Demand?}

Response case demand exhibits a strong seasonal variation. As was previously discussed, summer months show a significant increase in the number of incidents, while the remainder of the year is relatively consistent. Figure 3.7 provided a multiyear heat map of SISAR incidents. Over multiple years, incident levels increase significantly between May and September and drop significantly outside of this time frame. The CCG experiences a peak in SAR incidents during the month of July (829 \pm 121 incidents) and a low during the month of January (227 \pm 29 incidents). 

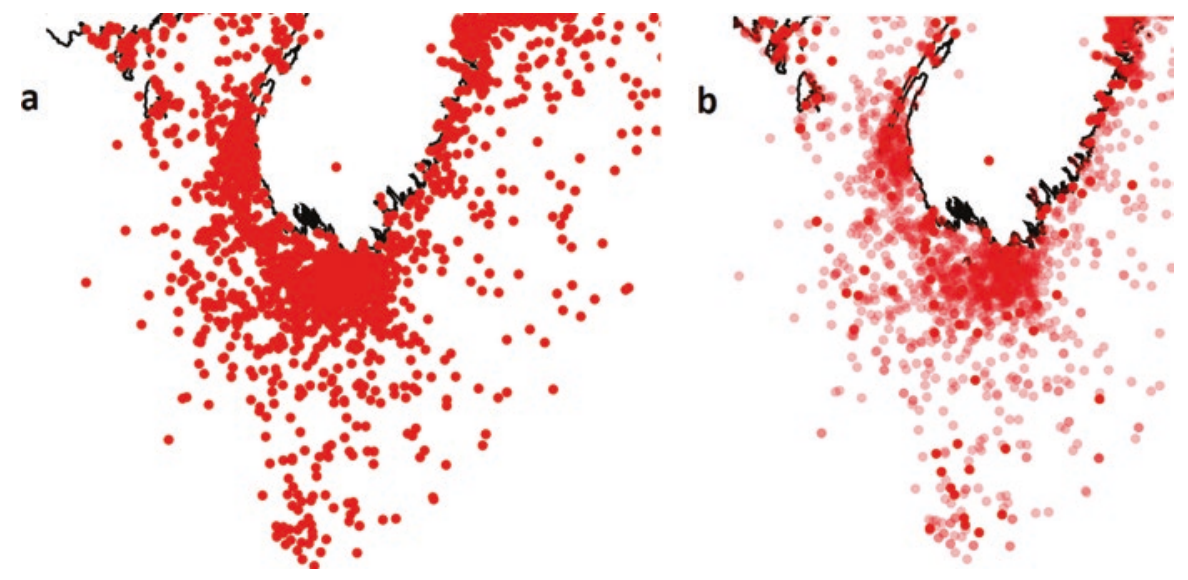

Fig. 3.14 (a) Spatial distribution of SAR incidents, (b) Spatial distribution of SAR incidents with opacity determined using incident severity (Eq. 3.1)

\subsubsection{What Areas Show a High Concentration of Most Severe Incidents?}

Understanding the spatial distribution of incidents is critical in planning emergency response operations and the allocation of resources (Marven et al. 2007; Akbari et al. 2017; Malik et al. 2011). Resources are often pre-positioned in areas with a higher concentration of SAR incidents to minimize response times and ultimately reduce the cost of providing lifesaving services. SISAR data provides a rich source of historical geo-referenced SAR incident data. The challenge is producing a visualization that allows the user to understand the significance of areas of higher SAR incident concentration. A visual analytic was created that uses the severity of incidents to aid in the interpretation of incident spatial location data. Figure 3.14 shows how using incident severity to control the opacity of incident location markers improves the definition of areas of severe incidents. This also improves the localization of hot spots, where more severe incidents are tightly grouped.

\subsection{Discussion}

Under-reporting in historical SAR incident databases can significantly affect the outcome of quantitative analyses, potentially leading to poor decision outcomes (Psarros et al. 2010). Under-reporting in the SISAR database used for this study could have a significant impact on the results discussed in Sect. 3.5. Under-reporting in SISAR is known to the CCG and believed to be largely due to technical 
challenges and human errors in constructing the database (Government of Canada 2019a). The CCG has determined the total missing cases, by region from 2006 to 2010: Quebec 0.9\%, Newfoundland and Labrador 2.9\%, Central and Arctic 2.7\%, Maritimes $4.6 \%$, and Pacific 30.9\%. Furthermore, to mitigate bias concerns related to the missing cases, a CCG evaluation team examined a sample of the missing cases and concluded that the missing cases are not biased (ibid). The fact that the total missing cases have been measured and that there was no bias in the missing cases helps to ensure that the SISAR database remains a primary data source supporting SAR incident data analysis in Canada.

Several times in this chapter we mentioned the USCG visual analytic tool cgSARVA (Malik et al. 2011), which focused on USCG historical response operation incident data in the Great Lakes region from 2002 to 2011. The Great Lakes region represents a major inland waterway where SAR is a shared responsibility between two nations. Canada and the United States have a long history of providing cross-border SAR in this region. It would seem reasonable that a complete assessment of maritime response, asset allocation, and risk assessment should reflect the cooperative nature of SAR in the Great Lakes region.

The inclusion of Canadian SAR stations and incident data in cgSARVA would undoubtedly influence the risk profiles generated by cgSARVA and reported in Malik et al. (2012). The generation of updated risk profiles that account for Canadian data could provide insight into the benefits of enhanced cross-border SAR coordination. Looking more broadly, maritime search and rescue in the Arctic also demands a high degree of international coordination and collaboration. The great importance of cooperation among Arctic nations in conducting SAR operations in the North is detailed in the Agreement on Cooperation on Aeronautical and Maritime Search and Rescue in the Arctic (Arctic Council 2011). The pooling of historical incident data and SAR resource locations and capabilities from all the parties to the Agreement could enable a more comprehensive analysis of SAR capabilities in the region and ultimately improve the delivery of SAR services.

Many researchers are also now starting to link environmental conditions and accident data to add context to historical SAR incidents. Wu, Pelot, and Hilliard (2009) have studied the influence of weather conditions on the relative incident rate of fishing vessels in Atlantic Canadian waters. Their analysis considered the following environmental factors: wave height, sea surface temperature, air temperature, ice concentration, fog presence, and precipitation. Ice concentration was shown to have the greatest influence on the magnitude of the relative incident rates for fishing vessels. In areas with low ice concentration, wave height was associated with higher incident rates. The presence of fog and precipitation was found to not be an important influence on relative incident rate.

More recently, Rezaee, Pelot, and Ghasemi (2016) extended the analysis of Wu, Pelot, and Hilliard (2009) and determined that the influence of weather conditions on relative incident rates in Atlantic Canadian waters is also largely dependent on vessel length. Similar studies have been conducted in other maritime areas. Goerlandt et al. (2017) examined navigational shipping accidents in the northern Baltic Sea area, successfully integrating accident data and environmental 
conditions. Atmospheric and sea ice data were used to reconstruct the navigational conditions that existed at the time of the accident to contextualize the incident, aimed at improving wintertime maritime transportation risk analysis.

Lastly, SAR response time estimation is receiving increasing attention in the literature and is a key factor in determining optimal SAR station location and assignment of SAR units. Siljander, Venalainen, Goerlandt, and Pellikka (2015) applied GIS-based tools and methods to evaluate SAR response time, considering environmental conditions. Their analysis considered the capabilities of the SAR unit and prevailing wave conditions at the time of the incident to improve estimates of response time for use in strategic SAR planning. SAR response times can be significant in the remote areas of the northwest Atlantic and Arctic. Not only are these regions remote, the environmental conditions can be very harsh, further increasing SAR response times. New methods are required to improve estimates of ship speeds in adverse environmental conditions, such as sea ice conditions, to improve the accuracy of response time estimation.

\subsection{Conclusion}

The CCG has collected data and information about SAR incidents involving CCG assets and personnel since the 1980s. This data provides CCG personnel with necessary information to support SAR planning, management, and operations. This rich SAR incident dataset provides researchers and analysts with a multivariate dataset that can be used to support a wide range of analysis and visualizations. In this chapter we discussed the use of SISAR incident data to explore temporal and spatial trends in the data using interactive web visualization techniques. The most prominent trends observed in the data included the increase in SAR incidents during the summer months, predominately on weekends and in the evening hours between $6 \mathrm{pm}$ and $11 \mathrm{pm}$.

In addition to temporal trends, the spatial location of SAR incidents was also examined. By plotting the coordinates for each SAR event on top of a map projection of the Canadian eastern coastline, it was possible to identify areas of high concentration of incidents. In this chapter we highlight the high concentration of incidents off the southern tip of Nova Scotia (see Fig. 3.14). In addition to plotting the location of the incident, the severity of the incident was used to create a visual analytics to control the opacity of the incident location point symbol (red circle). The use of opacity was effective in refining regions of higher concentration of severe incidents.

These interactive visualizations were effective in identifying several temporal and spatial patterns in the SISAR data. These visualizations could be used by an analyst to support decisions regarding SAR station manning, SAR station location, and employee shift scheduling (monthly, daily, and hourly). The continued use of SISAR data to improve decision-making in the CCG will help to ensure the delivery of SAR services is cost-effective and that response times are minimized, ultimately saving more lives. 


\section{References}

Akbari, A., Eiselt, H., \& Pelot, R. (2017). A maritime search and rescue location analysis considering multiple criteria, with simulated demand. INFOR: Information Systems and Operational Research, 56(1), 92-114.

Akbari, A., Pelot, R., \& Eiselt, H. (2018). A modular capacitated multi-objective model for locating maritime search and rescue vessels. Annals of Operations Research, 267(1), 3-28.

Arctic Council. (2011). Agreement on Cooperation on Aeronautical and Maritime Search and Rescue in the Arctic. 12 May 2011; Entered into force in 2013.

Bostock, M. (2019). D3js data-driven documents. https://d3js.org/. Accesed 28 June 2019.

Bostock, M., Ogievetsky, V., \& Heer, J. (2011). $\mathrm{D}^{3}$ data-driven documents. IEEE Transactions on Visualization and Computer Graphics, 17(12), 2301-2309.

David, A., \& Tauro, C. (2015). Web 3D data visualization of spatio temporal data using data driven document (D3js). International Journal of Computer Applications, 111(4), 42-46.

ESRI ArcGIS. (n.d.) Albers equal area conic projection (ArcMap 10.7). http://desktop.arcgis.com/ en/arcmap/latest/map/projections/albers-equal-area-conic.htm. Accessed 2 July 2019.

Goerlandt, F., Venäläinen, E., \& Siljander, M. (2015). Utilizing geographic information systems tools for search and rescue capacity evaluation. Scientific Journals of the Maritime University of Szczecin, 43(115), 55-64.

Goerlandt, F., Goite, H., Valdez Banda, O. A., Höglund, A., Ahonen-Rainio, P., \& Lensu, M. (2017). An analysis of wintertime navigational accidents in the northern Baltic Sea. Safety Science, 92, 66-84.

Government of Canada. (2003). Alerting, detection, and response: Dealing with accidents at sea. St. John's: Fisheries and Oceans Canada.

Government of Canada. (2019a). Canadian Coast Guard search and rescue and Canadian Coast Guard Auxiliary evaluation report. Fisheries and Oceans Canada. https://www.dfo-mpo.gc.ca/ ae-ve/evaluations/11-12/SAR-CCGA-eng.htm\#2.1. Accessed 25 June 2019.

Government of Canada. (2019b). Search and rescue posture review 2013. Department of National Defence. https://www.canada.ca/en/department-national-defence/corporate/reports-publications/search-and-rescue-posture-review-2013.html. Accessed 3 July 2019.

Government of Canada. (2019c). Maritime search and rescue in Canada. Canadian Coast Guard. http://www.ccg-gcc.gc.ca/eng/CCG/SAR_Maritime_Sar. Accessed 25 June 2019.

Malik, A., Maciejewski, B., Maule, B., \& Ebert, S. (2011). A visual analytics process for maritime resource allocation and risk assessment. In 2011 IEEE conference on Visual Analytics Science and Technology (VAST) (pp. 221-230). Providence: Institute of Electrical and Electronics Engineers.

Malik, A., Maciejewski, R., Jang, Y., Oliveros, S., Yang, Y., Maule, B., et al. (2012). A visual analytic process for maritime response, resource allocation and risk assessment. Information Visualization, 13(2), 93-110.

Marven, C. A., Canessa, R. R., \& Keller, P. (2007). Exploratory spatial data analysis to support maritime search and rescue planning. In J. Li, S. Zlatanova, \& A. G. Fabbri (Eds.), Geomatics solutions for disaster management (pp. 271-288). Berlin/Heidelberg: Springer.

Murray, S. (2013). Interactive data visualization for the web: An introduction to designing with D3. Sebastopol: O'Reilly Media.

Pelot, R., \& Plummer, L. (2008). Spatial analysis of traffic and risks in the coastal zone. Journal of Coastal Conservation, 11, 201-207.

Rezaee, S., Pelot, R., \& Ghasemi, A. (2016). The effect of extreme weather conditions on commercial fishing activities and vessel incidents in Atlantic Canada. Ocean \& Coastal Management, $130,115-127$.

Shahrabi, J. (2003). Spatial and temporal analysis of maritime fishing and shipping traffic incidents. PhD dissertation, Dalhousie University. 
Siljander, M., Venäläinen, E., Goerlandt, F., \& Pellikka, P. (2015). GIS-based cost distance modelling to support strategic maritime search and rescue planning: A feasibility study. Applied Geography, 57, 54-70.

Sonninen, M., \& Goerlandt, F. (2015). Exploring the context of maritime search and rescue missions using visual data mining techniques. Scientific Journals of the Maritime University of Szczecin, 43(115), 79-88.

United States Department of Homeland Security. (2018). Snapshot: How Coast Guard response is benefitting from S\&T's university partnerships. https://www.dhs.gov/science-and-technology/ news/2018/01/30/snapshot-how-coast-guard-response-benefitting-st-s-university. Accessed 25 June 2019.

Van Wijk, J., \& Selow, S. (1999). Cluster and calendar based visualizations of time series data. In Proceedings of the 1999 IEEE symposium on information visualization, San Francisco, CA, USA, 1999, 4-9.

Ware, C. (2004). Information visualization: Perception for design. San Francisco: Elsevier.

Wu, Y., Pelot, R. P., \& Hilliard, C. (2009). The influence of weather conditions on the relative incident rate of fishing vessels. Risk Analysis, 29(7), 985-999.

Open Access This chapter is licensed under the terms of the Creative Commons Attribution 4.0 International License (http://creativecommons.org/licenses/by/4.0/), which permits use, sharing, adaptation, distribution and reproduction in any medium or format, as long as you give appropriate credit to the original author(s) and the source, provide a link to the Creative Commons license and indicate if changes were made.

The images or other third party material in this chapter are included in the chapter's Creative Commons license, unless indicated otherwise in a credit line to the material. If material is not included in the chapter's Creative Commons license and your intended use is not permitted by statutory regulation or exceeds the permitted use, you will need to obtain permission directly from the copyright holder.

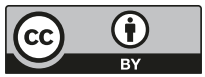

